time, as the apparatus stood for days continuously evacuated.

These results and others led to the conclusion that the hydrogen ions came not from $\mathrm{HCl}$ molecules but from $\mathrm{H}_{2}$ molecules formed probably by some process requiring the presence of the hot filament and of water vapour given off by the walls of the tube. Furthermore, the arrangement of the electrodes and fields in the tube permitted the discovery that the $\mathrm{Cl}$ - ions observed came from close to the filament and that none came from any region in which electrons of more than three or four volts energy were available for impact. It thus seems possible not only to account for the existence of the $\mathrm{H}^{+}$and $\mathrm{Cl}$ ions but also to eliminate them definitely as the products of inelastic electron impacts of 13.8 or more volts with $\mathrm{HCl}$ molecules.

A detailed report of the experiments is being prepared for publication.

Henry A. Barton (National Research Fellow).

Jefferson Physical Laboratory, Harvard University,

Cambridge, Mass., U.S.A., Dec. 16.

\section{A New Pressure-Temperature Formula for Vapours.}

AвоUT forty formulæ have been proposed to represent the relation between the pressure $p$ and temperature $t$ of saturated vapours. The suggested formulæ reproduce the experimental results only on a limited part of the vapour-pressure curve, and not on the whole of it. Many of them give inexact results at very low temperatures or in the proximity of the critical point. Finally, none of them reproduces the critical point as the singular point (end-point, le point d'arrêt) of the pressure-temperature curve.

I have succeeded in finding a formula which satisfies all the claims both in respect to precision and in respect to correct reproduction of the conditions on the limits.

The formula may be written as follows :

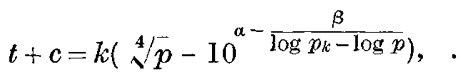

where $p_{k}$ is the critical pressure, and $c, k, a, \beta$ are constants, different for different substances.

At pressures $p$ sufficiently high (for ordinary liquids, throughout the range from $1-3$ atmospheres up to the critical pressure), the difference $\log p_{k}-\log p$ is small. The exponent $a-\frac{\beta}{\log p_{k}-\log p}$ is therefore a negative number with a large absolute value, so that the exponential term practically does not differ from zero. The formula thus becomes simply

$$
t+c=k \sqrt[4]{p} .
$$

Such a linear relation between $t$ and $\sqrt[4]{p}$ was found in 1883 by Jarolimek.

At $p=p_{k}, t$, as given by the formula (1), changes discontinuously; thus the formula reproduces the critical point. When $p$ is extremely small (therefore at very low temperatures) the term $\sqrt[4]{p}$ disappears in comparison with the exponential term, and the formula becomes

$$
\log p=\log p_{k}-\frac{\beta}{a-\log \left(-\frac{t+c}{k}\right)} .
$$

Formula (1) represents with sufficient accuracy the experimental data for the most varied substances; for example, for liquid metals (also for mercury in the whole explored interval from $0^{\circ}$ to $880^{\circ}$ ), for organic liquids studied by $\mathrm{S}$. Young, and for permanent gases.

The constant $c$ has usually the values lying between 0 and 273. Helium shows a remarkable peculiarity : it has $c=273$; thus, for helium the fourth root of the vapour pressure, in virtue of formula (2), appears to be approximately proportional to the absolute temperature.

Water, as usual, shows an anomaly: the index of the root is here $13 / 3$ instead of 4

A detailed account will be published in one of the physical journals.

Thermotechnical Institute, Moscow, Dec. 18.

\section{'Hard Seeds' in Leguminosæ.}

IT may be of interest to Mr. Alexander Nelson and to other readers of NATURE (Dec. 4, 1926, p. 804) to know that sulphuric acid has been used industrially for the promotion of germination in hard leguminous seeds for some years. The substitution on an extensive scale of Indigofera arrecta for the previously cultivated Indigofera sumatrana by the indigo planters of Bihar during the early years of the century was, in fact, made possible largely by the introduction of the sulphuric acid method of seed treatment into their agricultural practice. The seed of Indigofera arrecta grown in Bihar was found to possess a very hard coat and to fail to germinate almost entirely under the conditions of soil moisture prevailing in northern India. Treatment with sulphuric acid led to perfect germination in a well-ripened sample of seed, and the method was used for many years and is, I believe, still used by the Bihar planters. The procedure differs slightly, however, from that indicated by Mr. Nelson in that concentrated, instead of dilute, acid is employed. A full description of the method as used in Bihar may be found in my report to the Bihar Indigo Planters' Association for the year 1906-7.

I reported the successful application of the procedure to other hard seeds, those of a series of fibreyielding jungle plants not exclusively Leguminosæ, in collaboration with R. S. Finlow in the Proceedings of the Asiatic Society of Bengal (New Series, vol. 3, No. 10) in 1907. Some of these seeds withstood the action of the concentrated acid for surprisingly long periods and germinated satisfactorily after it.

In the same year a paper in which an attempt was made to explain the cause of hardness in the seeds of Indigofera arrecta was published by D. L. Day and myself (Annals of Botany, vol. 21, No. 81). Our findings, as regards hardness being due to a surface deposition on the seed-coat, were in accordance with those of Mr. Nelson; we were, however, unable to determine the precise nature of the coating. Information on this point will be most interesting.

The Biological Station,

Nanaimo, B.C., Dec. 27.

Television.

As Mr. J. L. Baird, in Nature of Jan. 29, mentions my name in connexion with 'television,' it may be of interest if I give a brief résumé of the demonstrations Mr. Baird has given to me at Motograph House. Last June he gave a demonstration of the trans. mission of the images of living people, showing gradations of light, shade, and detail. An account of this demonstration was published in NATURE of July 3. 